AperTO - Archivio Istituzionale Open Access dell'Università di Torino

\title{
Object temporal connotation
}

\section{This is the author's manuscript}

Original Citation:

Availability:

This version is available http://hdl.handle.net/2318/2022

since 2015-12-25T16:04:22Z

Publisher:

Academic Press Incorporated:6277 Sea Harbor Drive:Orlando, FL 32887:(800)543-9534, (407)345-4100,

Terms of use:

Open Access

Anyone can freely access the full text of works made available as "Open Access". Works made available under a Creative Commons license can be used according to the terms and conditions of said license. Use of all other works requires consent of the right holder (author or publisher) if not exempted from copyright protection by the applicable law. 


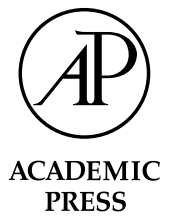

\title{
Object temporal connotation
}

\author{
Cristina Becchio $^{\mathrm{a}, *}$ and Cesare Bertone ${ }^{\mathrm{b}}$ \\ ${ }^{a}$ Center for Cognitive Science, University and Polytechnic of Turin, Via Po 14, Torino 10123, Italy \\ ${ }^{\mathrm{b}}$ Centro di Ontologia Teorica e Applicata, University of Turin, Torino, Italy
}

Accepted 1 April 2003

\begin{abstract}
Recent studies show that what connotes an object is first of all a certain spatio-temporal structure. In this paper we describe some of the temporal features characterizing the temporal structure of objects: pre-existence, persistence, conservation of identity in spite of perceptive discontinuity, surviving changes in colour, size, and shape. We argue that time is an indispensable attribute for every type of object and briefly discuss the implication of this view with respect to a specific neuropsychological syndrome: unilateral spatial neglect.
\end{abstract}

(C) 2003 Elsevier Science (USA). All rights reserved.

Keywords: Object; Temporal features; Time; Permanence

\section{The spatio-temporal structure of objects}

What is an object? What counts as an object?

Some researchers, such as David Marr, expressed a pessimistic opinion about the possibility of providing an answer to this question:

Is a nose an object? Is a head one? Is it still one if it's attached to a body? What about a man on horseback? These questions show that the difficulties in trying to formulate what should be recovered as a region from an image are so great as to amount almost to philosophical problems. There is really no answer to them - all these things can be an object if you want to think of them that way, or they can be part of a larger object (Marr, 1982).

When considering the mind as a whole, Marr's pessimism is certainly appropriate: almost everything can be conceived as an object. According to the Oxford English Dictionary, object is "something placed before the eyes, or presented to the sight or other sense; an individual thing seen or perceived, or that may be seen or perceived; a material thing." Mountains and trees, but also quarks, chairs and parliaments: all are objects.

At the earlier levels of sensorial analysis and with regard to mental processes as visual attention, the

\footnotetext{
${ }^{*}$ Corresponding author. Fax: +11-8159039.

E-mail address: becchio@psych.unito.it (C. Becchio).
}

questions raised by Marr admit, however, a precise answer. Evidence from different experimental paradigms (change detection task, selective looking, multiple object tracking) demonstrate that what connotes an object in general is first of all a certain spatio-temporal structure (Jiang, Olson, \& Chun, 2000; Pylyshyn, 2001; Quinlan, 1998; Sagi \& Julesz, 1985; Scholl, 2001).

Recent studies have explored what kind of spatial features a stimulus must have in order to count as an object. In the context of the multiple object tracking method-a paradigm that will be described in the following sections - Scholl, Pylyshyn, and Feldman (2001) have begun to examine some of the spatial factors which mediate the degree to which various clusters of features can count as an object. The results show a differential contribution of factors such as connectedness, part structure, and other types of perceptual grouping.

What remains to be defined in an experimental and structured way is the temporal structure of objects, that is the temporal features which connote objecthood.

In this paper we will try to show that time is not less problematic than space. A way to approach the problem is to consider a pile of sand and a pile-shaped object coated in sand. What is it that distinguishes them? Using the method of the violation of expectancy, Huntley-Fenner, Carey, and Salimando (2002) have 
shown how 8-month-old infants have permanence for a pile-shaped object, but not for a pile of sand. The study used pile-shaped objects and piles perceptually indistinguishable. It was only upon seeing the entity being poured (in the case of sand) or lowered (in the case of the object) onto the stage that infants could identify the resulting pile-shaped entity as the sand or the sand object.

A pile of sand is not an object, in spite of the fact that when stationary it may be perceptually indistinguishable from one. To comprehend why we must consider the temporal features which connote an object. In the next section, drawing on evidences from experimental psychology, neuropsychology and studies on young infants, we will describe some of the temporal features connoting objecthood. We will argue that, as shown by the experiment of Huntley-Fenner et al. (2002), spatio-temporal features have priority over surface properties such as shape and colour. In the last section, we will discuss the implication of these views with respect to a specific neuropsychological syndrome: unilateral spatial neglect.

\section{Temporal features of objecthood}

Time is an indispensable attribute for every type of object, be it visual or auditory. Contrary to spatial dimension, which seems to regard visual but not auditory objects, time connotes also auditory objects such as a melody (Kubovy \& Van Valkenburg, 2001).

In this section we describe some of the temporal features connoting objecthood: pre-existence, persistence, and conservation of identity through occlusion.

\subsection{Pre-existence}

Upon entering a dark room and switching on the light, I have the sensation that the illuminated objects were already there. The apparition of the object in the perceptive field marks the beginning of the object's presence, but not the beginning of its existence: the object appears as pre-existent. This is the form of permanence that Michotte (1950) calls permanence of anteriority to indicate the fact that the object or one of its parts seem to have had an existence anterior to their perception.

In the phenomenon of the "screen effect," where an object is seen to emerge from behind an occluder, the pre-existence turns into the impression of "coming out from." "When the speed and the size are conveniently chosen, the observer," writes Michotte (1950), "doesn't see the bar born and grow up, as it actually does, but sees a rigid bar of fixed dimension, appear from the square, slide from beneath the square, that formerly would have covered it."
The bar pre-exists: although unperceived, it exists before its apparition.

\subsection{Persistence}

On switching off the light, objects disappear without going out of existence. They survive their disappearance, continuing to exist even when they ceased to be visible (permanence of posteriority). Recent studies (Spelke, Kestenbaum, Simons, \& Wein, 1995), based on the paradigm of the violation of expectancy, show how 4.5 month-old infants expect objects to continue to exist when out of view. Infants were shown two screens with a gap between them. A first object emerged from the left edge of the left screen and then returned behind the screen; after a suitable delay, a second, physically identical object emerged from the right edge of the right screen and then returned behind it. No object ever appeared in the space between the two screens. Like adults, infants expected to find two distinct objects behind the two screens.

Since only one object could be seen at a time, to expect two distinct objects infants must have maintained both objects when out of view. These results suggest that infants have the permanence of objects and interpret the spatio-temporal discontinuity as evidence for two numerically distinct objects.

\subsection{Conservation of identity in spite of perceptive discontinuity}

Michotte (1950) defines this feature permanence of continuity: the object remains itself, maintains its identity, in spite of its discontinuous perceptive presence.

A useful paradigm for studying the maintenance of objects over time is the multiple object tracking (MOT), thought up by Pylyshyn (Pylyshyn \& Storm, 1988). In the canonical MOT experiment, subjects view a display consisting of a field of identical items (bidimensional stimuli such as points, plus signs or circles). A certain subset of items is flashed to mark it's status as target. All the items then begin to move independently and unpredictably about the screen. Subjects must attentionally track the targets. Since targets and distractors are identical during the motion phase, subjects can only succeed by picking out the targets when they are initially flashed and keep track of them. A large number of experiments have shown that observers can individuate and keep track on up to five targets in a field of 10 identical elements.

In a series of three MOT experiments, Scholl and Pylyshyn (1999) found that subjects are able to successfully keep a track of the items even when they are briefly, but completely occluded during their motion. The experiments evaluated different conditions of spatio-temporal interruption: occlusion, instantaneous disappearance and reappearance, implosion and explo- 
sion. In the occlusion condition the display included two outlined rectangles acting as occluders: items disappeared and reappeared from behind them, being absent from the display during their motion to the degree that they intersected one of these occluders. In the disappearance and reappearance condition items disappeared and reappeared instantaneously. In the implosion and explosion condition disappearance and reappearance were again gradual, but from the centres of the items: instead of accreting and deleting along a fixed contour, as in the occlusion condition, items imploded and exploded into and out of existence from their centres.

The results show that successful tracking requires the presence of accretion and deletion cues along a fixed contour: performance is severely impaired when the items appear and disappear in ways which do not implicate the presence of occluders, as in the instantaneous disappearance/reappearance condition and implosion/ explosion condition.

The existence of a mechanism for tracking through occlusion is supported by neuropsychological evidence, discussed by Scholl and Pylyshyn. Assad and Maunsell (1995) have, for example, discovered in the posterior parietal cortex of the monkey groups of neurons which fire as though signaling the presence of motion behind an occluder, even during intervals in which there is no motion on the retinal image.

The possibility that these neurons are part of an object tracking system is supported by recent neuroimaging studies indicating increased parietal activation in a multiple object tracking task, relative to a passive viewing condition (Culham et al., 1998). In line with these results, Michel, Henaff, and Intriligator (1997) have found that patients with parietal brain lesions are severely impaired in attentive tracking tasks.

What are the implications of such results for permanence of continuity? According to the authors, this pattern of results suggests that there is a mechanism for the individuation and maintenance of visual objects that does not require spatio-temporal continuity, but has a specific tolerance for interruptions of presence consistent with occlusion (Scholl \& Pylyshyn, 1999).

Bower (1974) shows how the same distinction between occlusion and interruption of existence takes place in infants. The experiment compares infants' visual search for objects that disappear by shrinking down to nothing with their visual search for objects that disappear by progressive deletion along a boundary. Infants searched for the missing object in the latter case, but not in the former.

\subsection{Surviving changes in colour, size, and shape}

In some circumstances, spatio-temporal features seem to be more tightly coupled with the representation of objects than surfaced-based features such as shape or colour. Using the MOT paradigm, Scholl, Pylyshyn, and
Franconeri (submitted) have, for example, shown that successfully attending to the targets throughout the tracking phase results in the encoding of spatio-temporal properties such as location and direction, but not featural properties such as colour and shape: subjects are able to report the location and direction of movement of the tracked items, but not changes in colours and shape.

The claim that featural differences do not affect the object identity is further underscored by the phenomenon of the "tunnel effect" (Burke, 1952). The basic paradigm is to show adults an object going behind a screen, or into a tunnel, followed by another object emerging out the other side. Under some circumstances the object is perceived as persisting behind the occluder. Interestingly, the amodal completion-so called because the observers do not see the object behind the screen-is exclusively determined by spatio-temporal considerations (the speed of the object, the time behind the occluder, the relative sizes of the object to that of the occluder). The features of the objects play a minimal role in the tunnel effect. If we see a red ball going behind an occluder and a blue box emerging from the other side, we perceive it as the same object that has changed properties.

The priority of spatio-temporal features is supported by neuropsychological evidence from Balint syndrome. Among many different type of deficits, including spatial disorientation, optic ataxia, and impaired depth perception, patients affected by Balint syndrome show simultanagnosia, that is the inability to perceive more than one object at a time. Such patients fail even the simplest task which require them to compute a relation between two separate objects. Balint patients are, for example, unable to determine if two parallel lines are of equal lengths. However, when the two lines are simply connected by other lines at each end to form a single shape, they can tell whether the shape is a rectangle or a trapezoid (Holmes \& Horax, 1919). As regards the perception of visual features, often simultanagnosic patients report seeing the colours of each of the objects in the display float through the single object which they are perceiving (Humphreys, Cinel, Wolfe, Olson, \& Klempen, 2000), as if the perceived object were not tied to any particular set of visual features.

\section{Spatio-temporal structure vs. spatio-temporal informa- tion}

The discussion so far has focused on the structure of objects (What counts as an object? Which properties qualify objecthood?). A related question concerns object individuation: what kind of information do we use for decision about individuation and numerical identity of objects? 
$\mathrm{Xu}$ and Carey (1996) show that infants, although sensitive to object properties, do not use kind differences as a basis for object individuation. Imagine the following scenario. One screen is put on a puppet stage. A duck emerges from behind the screen and returns behind it. Then a ball emerges from behind the same screen and then returns. How many objects are behind the screen? For adults, the answer is at least two objects: a duck and a ball. As there is only one single screen occluding the objects, this answer does not rely on spatio-temporal evidence, but on considerations relating to object properties and kind. Shown the above event, 10-monthold infants fail to draw the inference that there should be two objects, whereas 12-month-old infants succeed in the task.

These results are consistent with the idea that, in adults, two distinct representational systems underlie object individuation (Carey \& Xu, 2001). The first is the so-called mid-level system (mid-level, in the sense that it falls between the low level sensory processing and high level categorial processing) that indexes attended objects and track them through time, without encoding their properties. This system, available since the first phase of infancy, privileges the spatio-temporal information. At around 12 months of age a second system of object individuation emerges, a fully conceptual system, drawing on kind information. Both systems are designed to solve similar problems: when the spatio-temporal information is lacking or ambiguous, object individuation is granted to this second system.

Returning to the question of the object structure, should be noted that both the mid-level system and the kind-based system lead to the identification of an object connoted by the above mentioned spatio-temporal features.

\section{Conclusions}

In psychology as in neuropsychology time has been generally regarded as a parameter to be varied, not as a topic of investigation.

In front of a proliferation of neuroscientific studies concerning the ways in which spatial locations are represented in egocentric (self-centred) and allocentric (other-centred) frames of reference, time has until now remained unexplored.

The consequences of this surprising lacuna are not incidental. The effects of a lack of investigation on time are, for instance, felt in the comprehension of neuropsychological pathologies such as unilateral neglect. Unilateral neglect is the name given to a collection of disorders in which the patients fail to perceive or respond to certain stimuli in their controlateral hemispace. Historically, the phenomena of neglect have been characterized exclusively in spatial terms, being analyzed with respect to different aspects of spatial cognition (for a review, see Kerkoff, 2001). But what about time?

In a famous experiment by Bisiach and Luzzati (1978), two patients with right hemisphere lesions were asked to imagine themselves at one end of a well-known square and describe all the places of business on the plaza. They failed to recall shops, cafes, etc., on the left. Remarkably, when imagining themselves at the other end of the plaza, they named the previously neglected places but omitted those recalled before.

An explanation of the deficit shown by these patients exclusively in spatial terms, fails to account for a paradoxical aspect: the previously recalled details were neglected and vice versa, the previously omitted details were recalled. Changing the vantage point disrupted the continuing existence of some details, which ceased to exist, whereas other details imploded into existence. How is this possible?

The temporal feature of permanence of anteriority and posteriority must, in some way, be disrupted in unilateral neglect. Neglect affects not only the unitarity of space, but the continuity of the temporal dimension. Patients affected by neglect not only fail to respond to stimuli presented in the hemispace controlateral to the lesion but behave as if the controlateral hemispace did not exist and had never existed.

The same considerations apply to object-based neglect. Many studies (see, for example, Caramazza \& Hillis, 1990; Driver, Baylis, Goodrich, \& Rafel, 1994; Subbiah \& Caramazza, 2000) suggest that neglect may also be object-based, in such a way that patients neglect halves of objects. Again, the neglected half is not simply out of sight, but has ceased to exist: patients behave as if it had never existed. As in the experiment by Bisiach and Luzzati (1978), the permanence of the object must in some way be disrupted.

\section{Acknowledgments}

This research has been supported by Ministry of Education (Quantitative and qualitative instruments for the analysis of the relationship in psychotherapy; Protocol No. 2001115311).

\section{References}

Assad, J. A., \& Maunsell, J. (1995). Neuronal correlates of inferred motion in primate posterior parietal cortex. Nature, 373, 518-521.

Bisiach, E., \& Luzzati, C. (1978). Unilateral neglect of representational space. Cortex, 14, 129-133.

Bower, T. G. R. (1974). Development of infancy. San Francisco, CA: W.H. Freeman.

Burke, L. (1952). On the tunnel effect. Quarterly Journal of Experimental Psychology, 4, 121-138. 
Caramazza, A., \& Hillis, A. (1990). Levels of representations, coordinate frames, and unilateral neglect. Cognitive Neuropsychology, 7, 391-445.

Carey, S., \& Xu, F. (2001). Infant knowledge of objects: Beyond object files and object tracking. Cognition, 80, 179-213.

Culham, J. C., Brandt, S., Cavanagh, P., Kanvisher, N. G., Dale, A. M., \& Tootell, R. B. H. (1998). Cortical fMRI activation produced by attentive tracking of moving targets. Journals of Neuropsychology, 80, 2657-2670.

Driver, J., Baylis, G., Goodrich, S., \& Rafel, R. (1994). Axis-based neglect of visual shapes. Neuropsychologia, 32, 1353-1365.

Holmes, G., \& Horax, G. (1919). Disturbances of spatial orientations and visual attention, with loss of stereoscopic vision. Archives of Neurology and Psychiatry, I, 385-407.

Humphreys, G. W., Cinel, C., Wolfe, J., Olson, A., \& Klempen, N. (2000). Fractionating the binding process: neuropsychological evidence distinguishing between binding of form binding of surface features. Vision Research, 40, 1569-1696.

Huntley-Fenner, G., Carey, S., \& Salimando, A. (2002). Objects are individuals but stuff doesn't count: perceive rigidity and cohesiveness influence infants' representation of small numbers of discrete entities. Cognition, 85, 203-221.

Jiang, Y., Olson, I. R., \& Chun, M. M. (2000). Organization of visual short-term memory. Journal of Experimental Psychology: Learning, Memory, and Cognition, 26, 683-702.

Kerkoff, G. (2001). Spatial hemineglect in humans. Progress in Neurobiology, 63, 1-27.

Kubovy, M., \& Van Valkenburg, D. (2001). Auditory and visual objects. Cognition, 80, 97-126.

Marr, D. (1982). Vision. New York: W.H. Freeman.

Michel, F., Henaff, M., \& Intriligator, J. (1997). Posterior callosum split and left parietal lesion reveal visual deficits in the right visual field. Cognitive Neuroscience Society Abstract.
Michotte, A. (1950). Á propos de la permanence phénoménale. Faits et théories. Acta Psychologica, 7, 298-322.

Pylyshyn, Z. (2001). Visual indexes, preconceptual objects, and situated vision. Cognition, 80, 127-158.

Pylyshyn, Z., \& Storm, R. W. (1988). Tracking multiple independent targets: Evidence for a parallel tracking mechanism. Spatial Vision, 3, 1-19.

Quinlan, P. T. (1998). The recovery of identity and relative position from visual input: Further evidence for the independence of processing of what and where. Perception \& Psychophysics, 60, 303-318.

Sagi, D., \& Julesz, B. (1985). What and where in vision. Science, 228, $1217-1219$.

Scholl, B. J. (2001). Objects and attention: the state of art. Cognition, $80,1-46$

Scholl, B. J., \& Pylyshyn, Z. (1999). Tracking multiple items through occlusion: clues to visual objecthood. Cognitive Psychology, 38, 259-290.

Scholl, B. J., Pylyshyn, Z., \& Feldman, J. (2001). What is a visual object? Evidence from target merging in multiple object tracking. Cognition, 80, 159-177.

Scholl, B. J., Pylyshyn, Z., \& Franconeri, S. L. (submitted). The relationship between property encoding and object based attention: evidence from multiple object tracking. Perception \& Psychophysics.

Spelke, E. S., Kestenbaum, R., Simons, D. J., \& Wein, D. (1995). Spatio-temporal continuity, smoothness of motion and object identity in infancy. British Journal of Developmental Psychology, $13,113-142$.

Subbiah, I., \& Caramazza, A. (2000). Stimulus-centered neglect in reading and object-recognition. Neurocase, 6, 13-31.

Xu, F., \& Carey, S. (1996). Infants' metaphysics: The case of numeral identity. Cognitive Psychology, 30, 111-153. 